\title{
Città del Vaticano, Biblioteca Apostolica Vaticana, Vat. gr. 176
}

\author{
I. e III. Costantinopoli, XIV sec.; II. [Costantinopoli], XIII sec.
}

Comp., ff. A, I-V, 1-193 (+ 99a, 189a, 192a), I', A'; i ff. A-A', perg., vergati in stile $\tau \tilde{\omega} v$

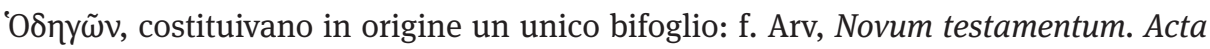

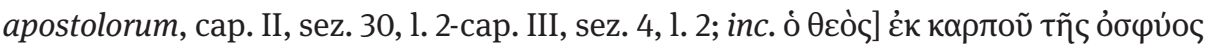

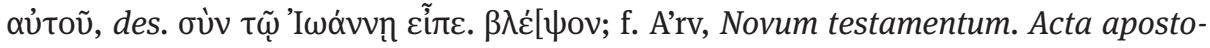

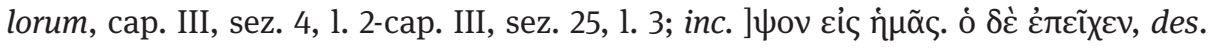

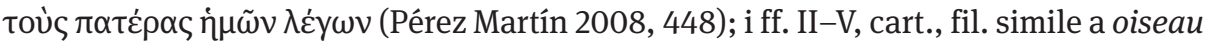
(Br nr. 12.250, 1566-1575), contengono il cosiddetto pinax Allacciano vergato da Giuseppe de Iuliis nel XVII secolo; sono bianchi i ff. 27v, 99a, 100r, 189, 192a. Legatura in pelle bianca con segnatura sul dorso.

Il codice consta di tre unità codicologiche: la prima e la terza, risalenti al XIV secolo, furono trascritte da Isacco Argiro e dall'Anonimo $\beta$; la seconda risale alla fine del XIII secolo ed è stata vergata da due copisti anonimi. La disposizione delle unità sembra rispondere ad un progetto editoriale avente come scopo l'allestimento di una miscellanea di testi di geografia e di teoria armonica: la prima sezione contiene una scelta pressoché completa dei capitoli teorici della Geographia di Tolomeo, corredata dagli scolii di Niceforo Gregora, cui segue un lungo scolio composto da Argiro ad una sezione del testo di Tolomeo (la paternità è resa esplicita nel titolo); la seconda e più antica unità reca gli Harmonica di Manuele Briennio ed è seguita dalla terza, con gli Harmonica di Tolomeo, accompagnati dai commentarii di Porfirio e Pappo.

\section{I. ff. 1-27 (Costantinopoli, terzo quarto del XIV sec.)}

Cart.; fil. ff. 1/2, 3, 5, 7 simile a ange (Br nr. 783, 1358-1359 o nr. 785, 1361), ff. 10-12, 14, 19, 21, 23, 26-27 simile a $\operatorname{arc}\left(\mathrm{Br} \mathrm{nr} .598,1357\right.$ o nr. 600, 1367 e 1361); $1-2^{8}(1 / 2-17), 3^{8+2}$ (27); num. dei fascicoli presente nel marg. inf. int. dei ff. 9v e 17v, numerati rispettivamente come $\alpha^{\prime}$ e $\beta^{\prime} ; \mathrm{mm} 272 \times 178=31 / /$ 186 // 31 / $24 \times 24$ // 110 // 11 / 27 / 6; a piena pagina, 11. 28/rr. 30, unità di rigatura mm 7 (f. 8r); rigatura a secco, tipi di rigatura differenti: fasc. I tipo S/L 21C1bn, (Muz 1-21/0-1/0/C); ff. 10-17 sul verso di ciascun foglio sono tracciate unicamente le rettrici; sistema di rigatura doppio: le linee orizzontali sono tracciate sul verso di ogni foglio e quelle verticali sul recto; nei ff. 26v-27r la disposizione dello spazio scrittorio cambia: a piena pagina, 11. 39, assenza di rigatura (f. 26v).

\section{Scrittura}

In questa sezione si alternano due copisti: Anonimo $\beta$ (ff. 1r-11v, 1. 28); <Isacco Argiro (Düring 1930, XxxIII; Mercati 1931, 229 n. 6)> (ff. 11v, 1. 29-27r). 


\section{Decorazione}

La decorazione, essenziale ed esigua, fu realizzata da Argiro, come pure i diagrammi geometrici associati al testo di Tolomeo (ff. 11r, 13r, 19v, 21rv, 26v).

\section{Contenuto}

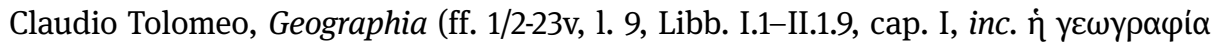

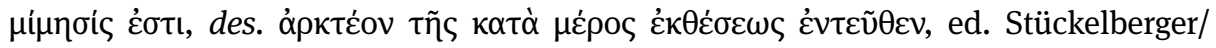

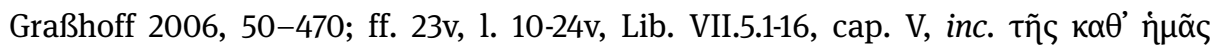

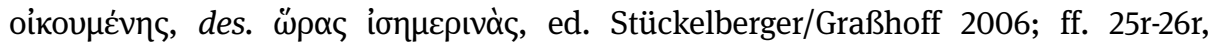

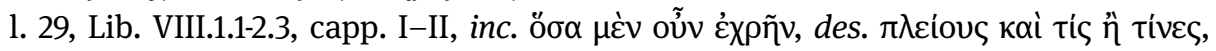

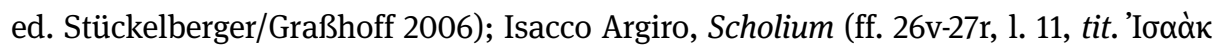

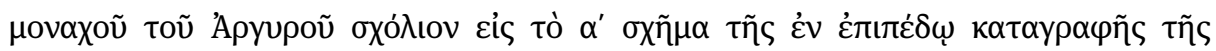

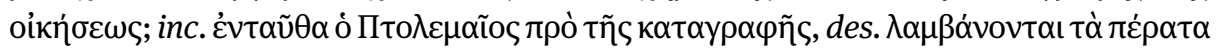

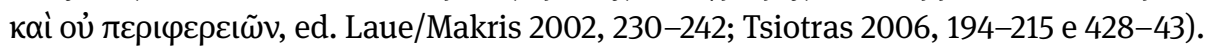

\section{II. ff. 28-99 (Costantinopoli ?, fine XIII sec.)}

Cart. orientale; $4^{6+1}(34), 5-11^{8}(90), 12^{6}(96), 13^{6-2}(99 a)$; nel marg. inf. del f. $51 \mathrm{r}$ resta traccia della numerazione del fascicolo, $\delta^{\prime}$ (in principio era un'unità codicologica a sé); mm $271 \times 185=13 / /$ 244 // $14 \times 18$ // 120 // 47; a piena pagina, ll. 33-38/rr. 33, unità di rigatura mm 7 (f. 33r); si intravedono le sole rettrici; foratura caduta con la rifilatura.

\section{Scrittura}

Nella sezione si distinguono tre mani: A (ff. 28r-30r, 1. 14); B (ff. 30r, 1. 15-ff. 99v, 1. 8); C (1l. 9-13 del f. 61v), dalla medesima educazione grafica, influenzate nell'esuberanza delle forme dalla cosiddetta Fettaugenmode, di cui tuttavia stemperano i toni più eccessivi.

\section{Decorazione}

L'ornamentazione è stata aggiunta da Argiro, che ha distinto le rubriche e i tituli di alcuni paragrafi, racchiudendoli tra due croci (ff. 37v, 45r, 50r, 52r, 55r, 57r, 60v, 61v, 68r, 71r, 76v, 80r, 88v, 90v, 92r, 95v, 96v). Anche la realizzazione degli schemi grafici e dei diagrammi geometrici (ff. 30r, 32rv, 37r, 46v, 47rv, 51v, 55r, 59v, 60r, 62r margine esterno, 63v, 64v, 65rv, 66rv, 67rv, 68rv, 69rv, 70rv, 71rv, 72rv, 73rv, 74rv, 75rv, 76rv, 
77v, 78rv, 79rv, 91v, 92v, 93rv, 94rv, 95rv, 96r, 98v) è opera di Argiro. Vi è una sola fascia ornamentale, formata da volute e racchiusa in un rettangolo, ad apertura della seconda unità (f. 28r).

\section{Contenuto}

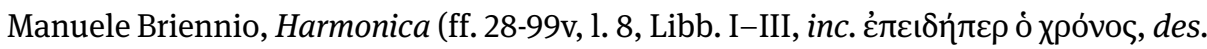

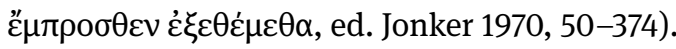

\section{III. ff. 100-192a (Costantinopoli, terzo quarto del XIV sec.)}

Cart.; fil. ff. 100-118 simile a arbalète (Br nr. 705, 1345 e nr. 706, 1323), ff. 121-165 simile a bélier (P XV.III, nr. 210 e nr. 212, 1360), f. 186 simile a poire (P XIV.II, nr. 583, 1360), ff. 178, 182, 185/8, 184/9 simile a bouc (P XV.III, nr. 374 e nr. 375, 1364); $14^{2+8}(109), 15-23^{8}(181), 24^{1+8}(189), 25^{4}(192 \mathrm{a})$; num. dei fascicoli non presente; $\mathrm{mm} 270 \times 188=13$ // 239 //18 $\times 21 / / 114$ // 53; a piena pagina, 11. 23-47/rr. 37, unità di rigatura mm 6 (f. 102r); tipo e sistema di rigatura non corrispondente ad alcuna codifica, giacché sono tracciate a secco sul verso di ogni foglio unicamente le rettrici; al centro dei ff. 179rv vi sono due riquadri rispettivamente di 11.22 e 11 . 11 di scrittura; la foratura non è presente; fil. simile a $\operatorname{arc}(\mathrm{Br}$ nr. 598, 1357 o nr. 600, 1367 e 1361).

\section{Scrittura}

Nell'unità si distingue un unico copista, identificato con < Isacco Argiro (Düring 1930, XXXIII; Mercati 1931, 229)> (ff. 100-192a).

\section{Decorazione}

Decorazione in inchiostro rosso di tituli, pinax (f. 100v) e incipit degli Harmonica di Tolomeo (f. 101r), preceduto da fasce decorative, di mano di Argiro.

\section{Contenuto}

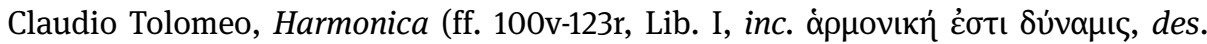

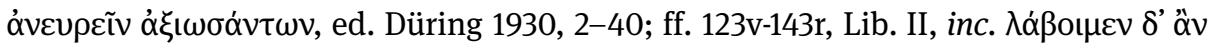

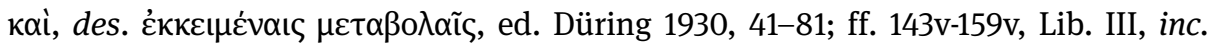

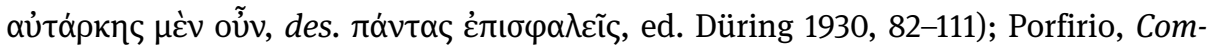

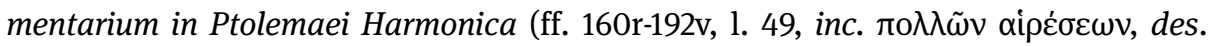

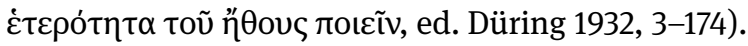




\section{Relazioni stemmatiche}

Per la Geographia di Tolomeo (ff. 1-27), il Vat. gr. 176 (Q) è apografo del Par. Coisl. 173 (r) (ff. 112-147), un manoscritto ampiamente postillato da Niceforo Gregora. Entrambi i testimoni appartengono alla famiglia $\rho$, il cui capostipite è il Marc. gr. Z. 516 (coll. 916) (R) (Diller, introd. a Nobbe, 1990, IX). In merito alla tradizione degli Harmonica di Manuele Briennio, che è stata ripartita da Jonker in due gruppi $a$ e $b$, il Vat. gr. 176 sembra collocarsi a metà tra le due famiglie (Jonker 1970, 44-47). Il Vat. gr. 176 costituisce una redazione a sé (A) degli Harmonica di Tolomeo: pur derivando testualmente sia da $f$ che da $m$, esso tramanda il capitolo II.14 integro (in quanto

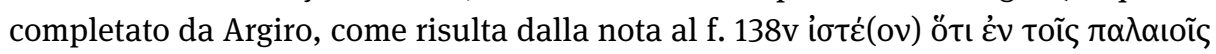

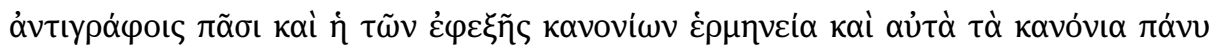

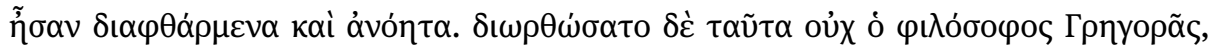

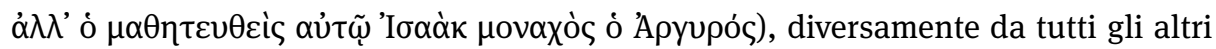
codici (Düring 1930, LXVI, LXXXVIII). Sono apografi diretti del Vaticano il Norimb. Cent. V App.38, copiato anch'esso da Argiro (Düring 1930, LXVI), il Vat. gr. 1411 e il Par.Suppl.gr. 449. Per quanto concerne il commentario di Porfirio, il suo testo differisce da altre recensioni bizantine e presenta numerose correzioni congetturali. $\grave{E}$ importante rilevare che il secondo titolo tramandato da Argiro sotto il nome di Pappo è in realtà frutto di un errore di lettura per TAYTOY presente nel modello (Düring 1932, XXVI); anche per questa porzione di testo il codice è antigrafo del Par. Suppl. gr. 449. Tra il 1541 e 1543 il codice fu utilizzato come modello da Nicola Murmure per gli scolii apposti alla stampa della Geographia tolemaica Scorial. 117.VII.19, di proprietà di Diego Hurtado De Mendoza. Tali scoli, di cui il Vat. gr. 176 è latore, recano i commenti alla Geographia di Niceforo Gregora e di Argiro (Caballero Sánchez 2014, 249-251; ed. Tsiotras 2006, 383-432).

\section{Storia del codice}

Il codice è presente nella Biblioteca Apostolica Vaticana dal pontificato di Sisto IV, come risulta dall'inventario del 1475 (Devreesse 1965, 59); nel XVII secolo, lo scriptor della Biblioteca Vaticana Giuseppe de Iuliis, coadiutore di Simone Porzio, lo munì del cosiddetto pinax Allacciano.

\section{Bibliografia}

\section{Cataloghi}




\section{Edizioni}

Stückelberger/Graßhoff (2006); Laue/Makris (2002); Jonker (1970); Düring (1930); Düring (1932).

\section{Codicologia e paleografia}

Bianconi (2008) 355 n. 57, 360 n. 73; Burri (2013) 12, 72, 74, 78, 86, 93, 96, 100, 126, 344, 348, 532, 538; Caballero Sánchez (2014) 247-251; Canart (2008a) 61; Estangüi Gómez (2013) 174; Mercati (1931) 229 e n. 6; Mondrain (2005) 20; Mondrain (2007) 67-68; Mountford (1926); Pérez Martín (2008) 446-447, 448; Tsiotras (2006).

\section{Riproduzioni}

Laue/Makris (2002) tav. 1; Pérez Martín (2008) tavv. 11-12. 